\title{
Funciones ejecutivas en el TDAH: una mirada a través del test de Rorschach
}

\author{
Cristina Fernández-Belinchón. Universidad Francisco de Vitoria \\ Laura Rodríguez-Moya. Hospital Universitario Fundación Alcorcón \\ José Carlos Peláez-Álvarez. Hospital Universitario Fundación Alcorcón \\ Recepción: 10 de Junio de 2017 | Aceptado: 16 de Junio de 2017 \\ Correspondencia: Cristina Fernández-Belinchón | Correo-e: cristina.fernandez@ufv.es
}

\author{
iD 0000-0002-3675-1370 \\ Citar: Fernández-Belinchón, C., Rodríguez-Moya, L. y Peláez-Álvarez, J. (2017). Funciones \\ ejecutivas en el TDAH: una mirada a través del test de Rorschach. ReiDoCrea, 6(2), 1-8.
}

\begin{abstract}
Resumen: El Trastorno de Déficit de Atención con Hiperactividad (TDAH) es uno de los trastornos más prevalentes y que mayores alteraciones genera en diferentes ámbitos. Sus síntomas principales son la hiperactividad, la inatención y la impulsividad, y se ha relacionado con otras problemáticas como el déficit en las funciones ejecutivas. Con este trabajo se pretende mostrar la importancia de realizar un buen diagnóstico y la relevancia del test de Rorschach para entender la complejidad del niño diagnosticado de TDAH. Se describen los principales resultados que las investigaciones han hallado en las áreas perceptiva, cognitiva, afectiva, interpersonal y de funcionamiento interno mediante el Rorschach en población infantil con TDAH. Los datos apuntan una confirmación del deterioro de determinados componentes de las funciones ejecutivas en los niños con el trastorno.
\end{abstract}

Palabras clave: TDAH | Funciones ejecutivas

Executive functions in ADHD: a look through the Rorschach Inkblot Test

Abstract: Attention Deficit Hyperactivity Disorder (ADHD) is one of the most prevalent disorders and it causes several problems in different areas. The main symptoms of the ADHD are hyperactivity, inattention and impulsivity, and the disorder has been related to other problems such as executive functioning deficit. This article aims to show the importance of making the correct diagnosis and the relevance of the Rorschach Inkblot Test to understand the complexity of the child with ADHD. We describe the main results that different research have found in the perceptive, cognitive, affective, interpersonal and internal functioning areas through Rorschach Inkblot Test in children with ADHD. The data confirm the deterioration of various components of executive functions in children with the disorder.

Keywords: ADHD | Executive functions

\section{Introducción}

El Trastorno por Déficit de Atención con Hiperactividad (TDAH) se considera en la actualidad la alteración más frecuente en la psicopatología infantil (González-Carpio y Serrano, 2016). La Asociación Psiquiátrica Americana (APA, 2014) aporta datos de prevalencia del $5 \%$ en niños y el $2,5 \%$ en adultos, en la mayoría de las culturas. En España, los estudios indican una prevalencia global entre el $4,6 \%$ y el $4,9 \%$ en niños entre 6 y 12 años (Cardo, Servera y Llobera, 2007; Jiménez, Rodríguez, Camacho, Alfonso y Artiles, 2015). Sin embargo, de manera subjetiva se sobrediagnostica el trastorno dentro de la escuela y la familia. Manga, González y Fournier, (2008) encontraron que los maestros describen como inatentos al $49 \%$ de los niños y al $27 \%$ de las niñas, lo que supera las cifras de prevalencia real, e indica lo disfuncional de la sintomatología conductual y la dificultad de manejo en el aula.

EI TDAH es un trastorno del neurodesarrollo y está compuesto principalmente por tres grandes síntomas: la hiperactividad o incapacidad para controlar el nivel de actividad motora, la inatención o conflicto para determinar qué estímulos son relevantes, y la 
impulsividad o déficit en la capacidad de reflexión previa a actuar (APA, 2014; Manga et al., 2008). Los niños diagnosticados de TDAH manifiestan también baja tolerancia a la frustración, dificultades para controlar los afectos y demorar la recompensa, y déficits en la planificación de acciones, en la inhibición conductual, en la regulación de la motivación y en la memoria de trabajo (Barkley, 1997, 2013; Orjales, 2011; Sanfeliu, 2011). Presenta además comorbilidad con otros trastornos externalizantes infantiles como el trastorno de conducta y el trastorno oposicionista o negativista desafiante (Manga et al., 2008; Meehan et al., 2008).

Es un cuadro complejo y su etiología se discute en la actualidad. Parece que en su manifestación influyen aspectos genéticos, biológicos, intrapsíquicos, familiares y sociales (Sanfeliu, 2011). Uno de los modelos más aceptados en relación al origen de la patología es el de Barkley (1997), quien lo considera un trastorno de la regulación interna y de la atención sostenida, secundario en muchos niños a déficits en la inhibición conductual, lo que se manifiesta en comportamientos impulsivos. Esa impulsividad dificulta prever las consecuencias de su conducta (Sanfeliu, 2011), generando problemas en cuidadores, profesores y familiares para planificar actividades y rutinas. Para Meehan et al. (2008), esta teoría de Barkley ofrecería una visión comprehensiva de los déficits en las funciones ejecutivas relacionados con el TDAH.

Esta alteración en las funciones ejecutivas es en estos momentos una de las hipótesis más estudiadas en relación al origen del trastorno. Se definen como un conjunto de procesos que incluyen la planificación, la organización, la flexibilidad cognitiva, la autorregulación, la memoria de trabajo, la inhibición de respuestas y la resolución de problemas, entre otras características (Anderson, 2002; Barkley, 1997; Barkley, Smallish y Fletcher, 2005; Berenguer, Roselló, Miranda, Baixauli y Palomero, 2016; Lezak, 1982).

Un funcionamiento correcto de las funciones ejecutivas permite al niño realizar conductas que facilitan la adaptación al entorno y un comportamiento exitoso e independiente (llonen et al., 2000). Sin embargo, aquellos individuos diagnosticados de TDAH presentan alteraciones en esta área, manifestando, entre otros síntomas, desinhibición conductual, impulsividad, ausencia de planificación y dificultades en la autorregulación.

Pero no todas las funciones ejecutivas están alteradas en el TDAH (Yáñez-Téllez y col., 2012), sino que existen déficits en algunos de sus componentes, como en la memoria de trabajo y en la inhibición, donde se ha encontrado un menor rendimiento en niños con el trastorno respecto a aquellos sin el diagnóstico (Berlin, Bohlin, Nyberg y Janols, 2004; Berenguer et al., 2016; Romero-Ayuso, Maestú, González-Marqués, Romo-Barrientos y Andrade, 2006; Schoemaker, Bunte, Andrews, Dekovié y Matthys, 2014). Sin embargo, para autores como Yáñez-Téllez y col. (2002), estas alteraciones no determinan la impulsividad y la hiperactividad de estos niños, sino que las deficiencias se encuentran en otros componentes, como el razonamiento abstracto y la flexibilidad cognitiva. Estos últimos autores hallaron en concreto en los niños con TDAH mayores errores y perseveraciones, y menor porcentaje de respuestas de nivel conceptual, mediante el test de clasificación de cartas de Wisconsin.

Estos déficits en la función ejecutiva tienen un impacto negativo en el establecimiento de relaciones interpersonales, en el funcionamiento familiar y en el rendimiento académico, debido a la dificultad que presentan en la planificación de objetivos y estrategias, lo que provoca problemas en la escuela y bajas calificaciones (Daley y Birchwood, 2010; González-Carpio y Serrano, 2016; Johnson y Reid, 2011; Manga et 
al., 2008). La relación entre las dificultades específicas de aprendizaje y el TDAH es cada vez más clara, desarrollando los niños problemas socioemocionales como consecuencia de fracaso en el aprendizaje o por una disfunción del sistema nervioso (Manga et al., 2008; Orjales, 2011).

Debido a las consecuencias del trastorno y el sufrimiento interno que genera, se hace imprescindible una correcta detección y valoración del mismo. El empleo de instrumentos que amplíen el conocimiento sobre estos sujetos, y no aporten información únicamente de los síntomas observables que presentan, permitiría un diagnóstico adecuado de la patología y ayudaría en la planificación de una intervención individualizada.

Este artículo pretende mostrar el funcionamiento de niños con TDAH en distintas áreas, entre ellas las funciones ejecutivas, y la relevancia del test de Rorschach como una herramienta eficaz en la detección de sintomatología del trastorno, que, si bien no debe ser empleado como única fuente de información, permite una mayor comprensión de sus características subyacentes (Cotugno, 1995). Para conseguir este objetivo se revisarán los estudios en los cuales se haya empleado el Rorschach en población infantil con diagnóstico de TDAH.

\section{TDAH y test de Rorschach}

El test de Rorschach es una prueba proyectiva perceptivo-cognitiva que define aspectos del funcionamiento de la personalidad del individuo, tanto la parte más situacional como la más estable, los recursos internos disponibles, la capacidad cognitiva, la afectividad, el área interpersonal, su autopercepción, y sus necesidades, actitudes y conflictos (Exner, 2003; Weiner, 2000). Presenta indicadores de impulsividad, de tolerancia al estrés, de la modulación de las descargas emocionales, del interés por los otros, de la prueba de realidad y de la exactitud perceptiva (Exner, 2001). Todo ello permite valorar a las personas de manera más profunda y global.

Está formada por 10 láminas con diferentes manchas que se caracterizan por su falta de estructuración, cinco de ellas en tonalidades negras y grises, dos láminas añaden a esos tonos el color rojo y en las tres últimas hay distintos colores. El sujeto tiene que responder libremente a la consigna de qué podría ser ese estímulo, y posteriormente indicar en qué zona de la mancha ha visto el objeto y qué le ha hecho pensar en eso (Exner, 2003).

El proceso de respuesta es complejo y la tarea requiere, además de habilidades perceptivas complejas, mediación verbal, traducción de las impresiones perceptuales (Meyer, 2016) e integración de funciones cerebrales visuales, semánticas y ejecutivas (Acklin y Wu-Holt, 1995). Para ello, intervienen procesos como la atención, la percepción, la codificación, el reconocimiento, la organización, la toma de decisiones y el análisis lógico; es decir, en el contexto de evaluación es necesario un correcto funcionamiento de las funciones ejecutivas y, dentro de ellas, la memoria de trabajo (Acklin y Wu-Holt, 1995; Weiner, 2003). Componentes que hemos señalado previamente de las funciones ejecutivas como la capacidad para formular metas, para planificar, para llevar a cabo los planes y ejecutarlos correctamente (Lezak, 1982), se ponen en marcha ante la estimulación ambigua que supone el Rorschach.

En la actualidad, se emplea como una prueba neuropsicológica y no sólo de personalidad, ya que se concibe como una tarea de resolución de problemas que muestra la actuación cognitiva habitual del sujeto (Erdberg, 2013; Muzio, 2016). Pietrowski fue el primer autor que en 1936 lo utilizó para diferenciar sujetos con 
alteraciones corticales orgánicas de otros individuos sin dicha lesión, observando 10 signos en el Rorschach que indicaban daño neurológico. Desde entonces, se ha ampliado el campo de estudios empíricos que relacionan la prueba con las neurociencias y que muestran su relación con otras medidas neuropsicológicas estandarizadas (Acklin y Wu-Holt, 1995; Meyer, 2016; Muzio, 2016; Selma, 2016).

Dentro del campo de la neuropsicología, desde hace dos décadas se comenzó a emplear la herramienta en niños con Trastorno por Déficit de Atención con Hiperactividad, con el objetivo de conocer sus recursos internos, su funcionamiento perceptivo, cognitivo e interpersonal, y su capacidad de autorregulación emocional. Los estudios no son abundantes, pero las conclusiones a las que se han llegado confirman el deterioro que existe en las funciones ejecutivas y en otras áreas, como la perceptiva, interpersonal y afectiva. A continuación se revisarán los principales resultados de las investigaciones empíricas en las cuales se ha comparado el funcionamiento de niños con TDAH frente a niños sin el trastorno, a través del test de Rorschach.

El primer estudio hallado versa de 1981, y fue llevado a cabo por Gordon y Oshman con una muestra de 40 niños con edades comprendidas entre los 6 y los 11 años. Los autores encontraron menos respuestas humanas completas $(H)$ y de movimiento humano (M), y más respuestas de animales completos $(A)$ entre el grupo de hiperactivos. Estos resultados indican una dificultad para emplear el razonamiento, la imaginación y la conceptualización, y un menor interés en las personas como seres humanos totales, reales y completos (Exner, 2001, 2003), lo que puede interferir en su rendimiento académico y el establecimiento de vínculos.

Bartell y Solanto (1995) por su parte coincidieron con Gordon y Oshman (1981) respecto a la disminución de $\mathrm{M}$ en 24 niños diagnosticados de TDAH, y hallaron, entre otros resultados, una peor calidad formal (aumento de X-\%), dato que señala la distorsión perceptiva que pueden experimentar. En ese mismo año, Cotugno (1995), con una muestra de niños de 5 y 6 años, halló un estilo más simplificador en la información compleja (aumento de Lambda), lo que genera que el niño ignore estimulación relevante del ambiente y tenga dificultades en la relaciones interpersonales, y una tendencia a ver la realidad de manera poco convencional y realista (disminución de $\mathrm{X}+\%$ y aumento de X-\% y Xu\%). Encontró además dificultades en la expresión de las emociones (disminución de Afr), un aumento del malestar interno (elevación de $\mathrm{SH}$ ), una menor autoestima y juicios más negativos sobre sí mismo (menor Índice de Egocentrismo), y una mayor ineficacia en la relación con el ambiente (aumento $\mathrm{CDI}$ ). Un dato importante y contradictorio respecto a las características que manifiestan los niños con TDAH, es que halló menor impulsividad (C) en el grupo de hiperactivos respecto al grupo control.

Más recientemente, Jain, Singh, Mohanty y Kumar (2005), empleando una muestra de 224 niños entre 6 y 11 años encontraron de nuevo menos respuestas $M$, peor calidad formal (disminución de $F+\%$ ) y menor contenido animal completo $(A)$ en aquellos con TDAH. Entre los resultados destacan la disminución de respuestas populares $(P)$, es decir, que las respuestas que pueden dar son menos convencionales y más individualistas que los niños sin el trastorno.

Finalmente, Meehan et al. (2008) hallaron en niños diagnosticados de TDAH entre 7 y 10 años unos resultados significativamente inferiores de movimientos humanos (M) y puntuaciones EA, lo que evidencia peor acceso a sus recursos personales internos, dificultando la organización de la información. Para los autores, esto puede interferir en 
su capacidad para demorar la gratificación, inhibir el comportamiento o regular su respuesta afectiva (Meehan et al., 2008).

Como conclusión general, se puede afirmar que el test de Rorschach explora las áreas perceptiva, afectiva, cognitiva e interpersonal del individuo, y puede captar la variabilidad individual en el funcionamiento, los recursos personales y la autorregulación. Los estudios han mostrado la capacidad de la prueba para confirmar el deterioro existente en determinados componentes de las funciones ejecutivas, pero no se centra exclusivamente en la valoración de estas alteraciones sino que también refleja el funcionamiento en el nivel perceptivo, emocional e interpersonal.

\section{Discusión}

El aumento de niños diagnosticados de TDAH vuelve imprescindible ampliar los conocimientos acerca de la patología. El diagnóstico se realiza habitualmente entre los 7 y los 9 años, pero uno o dos años antes los padres detectan la excesiva actividad motora del niño o la falta de continuidad en las tareas que realiza (APA, 2014; Romero-Ayuso y col., 2006). Se hace relevante un diagnóstico precoz y específico que facilite la orientación a la familia y la escuela, para poder reducir las consecuencias en la edad adulta (Romero-Ayuso y col., 2006).

Los resultados del test de Rorschach presentados coinciden en señalar que los niños con diagnóstico de TDAH muestran diferencias estadísticamente significativas respecto a aquellos sin el trastorno. Cognitivamente, presentan alteraciones en las funciones ejecutivas, lo que se observa en la prueba mediante variables que muestran sus dificultades en el razonamiento, la abstracción, la conceptualización de la información, la imaginación, la concentración y la recuperación de la representación interna de ellos mismos y los otros (Bartell y Solanto, 1995; Cotugno, 1995; Gordon y Oshman, 1981; Jain et al., 2005; Meehan et al., 2008). Se han hallado también capacidades positivas en ellos, en concreto, que poseen recursos internos para hacer frente a las demandas del medio, pero tienen dificultad para acceder a los mismos 0 los emplean de manera inadecuada (Meehan et al., 2008), lo que se traduce en un funcionamiento ineficaz con su medio (Cotugno, 1995).

Estos datos coinciden con los encontrados en otras investigaciones respecto al déficit que muestran estos niños en determinados componentes de las funciones ejecutivas, como la memoria de trabajo, el razonamiento abstracto y la inhibición (Berlin et al., 2004; Berenguer et al., 2016; Romero-Ayuso et al., 2006; Schoemaker et al., 2014; Yáñez-Téllez et al., 2002). Dichas alteraciones en las funciones ejecutivas son importantes como hemos visto para la planificación de las intervenciones escolares y psicoterapéuticas.

En el nivel perceptivo, existe una identificación errónea del campo estimular, que produce distorsión de ciertos aspectos de la realidad, respuestas menos convencionales y más individualistas que otros niños sin TDAH (Cotugno, 1995; Gordon y Oshman, 1981; Jain et al., 2005). Este modo de afrontar los estímulos puede generarles dificultades en la adaptación con sus iguales y conflictos en sus relaciones interpersonales.

$Y$ afectivamente, se ha hallado un aumento del malestar interno, una menor autoestima y dificultades para expresar las emociones (Cotugno, 1995). Estos datos confirman que los niños con TDAH padecen un alto sufrimiento emocional que repercute en el funcionamiento y la actuación (Orjales, 2011) y alto nivel de ansiedad (De la Peña et al., 2016). El deterioro observable en la capacidad de autocontrol es 
señal de la inmadurez en el control inhibitorio y en las funciones ejecutivas, así como de las deficiencias en el afrontamiento de problemas, tal como señaló Barkley (1997). El conjunto de síntomas les puede generar conflictos en la escuela y la familia.

Todos los datos presentados muestran que estos niños necesitan un apoyo motivacional de los adultos y el empleo de estrategias de enseñanza activa para conseguir aumentar su rendimiento y disminuir la frustración que sienten frente al fracaso (Berenguer y col., 2016). Es necesaria por tanto una intervención temprana tanto con pacientes como con familiares, con el objetivo de reducir los conflictos, reorientar las metas marcadas, y entender las necesidades de ambas partes (Cotugno, 1995).

Con este trabajo se ha pretendido mostrar la relevancia del test de Rorschach en el análisis del niño con diagnóstico de TDAH en su totalidad. Las herramientas diagnósticas neuropsicológicas disponibles se centran en la sintomatología observable, pero no aportan datos sobre lo que experimenta internamente el niño con el trastorno. Es importante comprender su manifestación, desde el malestar afectivo que siente el niño y la frustración que sus déficits le generan, y no tanto desde los síntomas manifiestos y los errores que comete. Sin embargo, y pese a todo lo descrito, nunca se debe emplear el Rorschach como única fuente de información de la sintomatología presentada por individuos con este diagnóstico, sino que debe ser un complemento de procedimientos psicométricos estandarizados (Cotugno, 1995).

La prueba se está empleando en baterías neuropsicológicas, y la colaboración entre todos los profesionales del Rorschach de diferentes países está permitiendo añadir datos neurofisológicos, conceptualizándola desde las neurociencias (Erdberg, 2013). Por eso, describir al niño a partir de sus necesidades, deseos y capacidades permitiría obtener mayores éxitos en el ámbito académico, interpersonal, familiar y personal.

La fortaleza de la presente revisión radica en la síntesis de los principales resultados hallados con niños con TDAH mediante el test de Rorschach, ya que en España no se han encontrado evidencias de que esto se haya realizado. Sin embargo, la mayor limitación es la insuficiencia de bibliografía encontrada que emplea la prueba en la valoración del niño con TDAH, lo que dificulta la generalización de conclusiones.

Por ello, nos planteamos como futura línea de investigación la realización de un estudio empírico empleando una muestra española de niños con diagnóstico de TDAH y sin él, utilizando pruebas neuropsicológicas y el test de Rorschach como herramientas. El objetivo será por un lado comparar los resultados con las investigaciones previas, y por otro valorar en primer lugar sus funciones ejecutivas y posteriormente el funcionamiento en el resto de áreas.

Referencias

Acklin, M.W., y Wu-Holt, P. (1995). Contributions of cognitive science to the Rorschach technique: cognitive and neuropsychological correlates of the response process. Journal of Personality Assessment, 67(1), 169-178.

American Psychiatric Association (2014). Manual diagnóstico y estadístico de los trastornos mentales DSM-5. Tomos 1 y 2. Madrid: Editorial Médica Panamericana.

Anderson, P. (2002). Assessment and development of executive functions (EF) during childhood. Child Neuropsychology, 8(2), $71-82$.

Barkley, R.A. (1997). Behavioral inhibition, sustained attention and executive functions: constructing a unifying theory of ADHD. Psychological Bulletin, 121(1), 65-94. 
Barkley, R.A. (2013). Taking charge of ADHD. The complete, authoritative guide for parents. New York: Guilford Press.

Bartell, S.S., y Solanto, M.V. (1995): Usefulness of the Rorschach Inkblot Test in assessment of attention deficit hyperactivity disorder. Perceptual and Motor Skills, 80(2), 531-541.

Berenguer, C., Roselló, B., Miranda, A., Baixauli, I., y Palomero, B. (2016). Funciones ejecutivas y motivación de niños con trastorno de espectro autista (TEA) y trastorno por déficit de atención con hiperactividad (TDAH). International Journal of Developmental and Educational Psychology, $1(1), 103-112$

Berlin, L., Bohlin, G., Nyberg, L., y Janols, L.O. (2004). How well do measures of inhibition and other executive functions discriminate between children with ADHD and controls. Child Neuropsychology, 10(1), 1-13.

Cardo, E., Servera, M., y Llobera, J. (2007). Estimación de la prevalencia del trastorno por déficit de atención e hiperactividad en población normal en la isla de Mallorca. Revista de Neurología, 44(1), 10-14.

Cotugno, A.J. (1995). Personality attributes of attention deficit hyperactivity disorder (ADHD) using the Rorschach Inkblot Test. Journal of Clinical Psychology, 51(4), 554-562.

Daley, D., y Birchwood, J. (2010). ADHD and academic performance: why does ADHD impact on academic performance and what can be done to support ADHD children in the classroom? Child: health, care and development, 36(4), 455-464.

De la Peña, C., Parra-Bolaños, N., Fernández Median, J. y Martínez Restrepo, O. (2016). Percepción del ajuste clínico en escolares con TDAH. Educación y Futuro Digital, 13, 23-34.

Erdberg, P. (2013). El Rorschach, Donald Winnicott y la neurociencia. Revista Española de Rorschach y Métodos Proyectivos, $26,64-71$.

Exner, J.E., Jr. (2001). Manual de codificación del Rorschach para el sistema comprehensivo. Madrid: Psimática.

Exner, J.E., Jr. (2003). The Rorschach: a Comprehensive System (4th ed.). New York: Wiley.

Fisher, M., Barkley, L.A., Smallish, L., y Fletcher, K. (2005). Executive functioning in hyperactive children as Young adults: attention, inhibition, response perseveration, and the impacto of comorbidity. Developmental Neuropsychology, 27(1), 107-133.

González-Carpio, G., y Serrano, J.P. (2016). Medication and creativity in Attention Deficit Hyperactivity Disorder (ADHD). Psicothema, 28(1), 2025

Gordon, M., y Oshman, H. (1981). Rorschach indices of children classified as hyperactive. Perceptual and Motor Skills, 52, 703-707.

Ilonen, T., Taiminen, T., Karlsson, H., Lauerma, H., Tuimala, P., Leinonen, K.M., Wallenius, E. y Salokangas, R. (2000). Impaired Wisconsin Card Sorting Test performance in first-episode severe depression. Nordic Journal of Psychiatry, 54(4), 275-280.

Jain, R., Singh, B., Mohanty, S., y Kumar, R. (2005). SIS-I and Rorschach diagnostic indicators of attention deficit and hyperactivity disorder. Journal of Projective Psychology and Mental Health, 12(2), 141-152.

Jiménez, J.E., Rodríguez, C., Camacho, J., Alfonso, M., y Artiles, C. (2015). Estimación de la prevalencia del trastorno por déficit de atención con 0 sin hiperactividad (TDAH) en población escolar de la Comunidad Autónoma de Canarias. European Journal of Education and Psychology, 5(1), $13-26$.

Johnson, J., y Reid, R. (2011). Overcoming executive function deficits with students with ADHD. Theory into Practice, 50, 61-67.

Lezak, M.D. (1982). The problem of assessing executive functions. International Journal of Psychology, 17, 281-297.

Manga, D., González, H., y Fournier, C. (2008). Trastornos por déficit de atención en la infancia. En A. Belloch, B. Sandín y F. Ramos, Manual de Psicopatología (Ed.rev.; vol. II) (pp.549-566). Madrid: McGraw Hill.

Meehan, K.B., Ueng-McHale, J.Y., Reynoso, J.S., Harris, B.H., Wolfson, V.M., Gomes, H., y Tuber, S.B. (2008). Self-regulation and internal resources in school-aged children with ADHD symptomatology: an investigación using the Rorschach inkblot method. Bulletin of the Menninger Clinic, 72(4), 259-282.

Meyer, G.J. (2016). Neuropsychologycal factors and Rorschach performance in children. Rorschachiana, 37(1), 7-27.

Muzio, E. (2016). Inkblots and neurons. Correlating typical cognitive performance with brain structure and function. Rorschachiana, 37(1), 1-6.

Orjales, I. (2011). Déficit de Atención con Hiperactividad. Madrid: CEPE. 
Pietrowski, Z. (1936). On the Rorschach method and its application in organic disturbances of the central nervous system. Rorschach Research Exchange, 1, 23-39.

Romero-Ayuso, D.M., Maestú, F., González-Marqués, J., Romo-Barrientos, C., y Andrade, J.M. (2006). Disfunción ejecutiva en el trastorno por déficit de atención con hiperactividad en la infancia. Revista de Neurología, 42(5), 265-271.

Sanfeliu, I. (2011). La hiperactividad. La acción inagotable. Madrid: Biblioteca Nueva.

Selma, H. (2016). Rorschach y la psicobiología de la personalidad. Universitas Psychological, 15(1), 39-64.

Schoemaker, K., Bunte, T., Andrews, K., Dekovié, M., y Matthys, W. (2014). Executive functions in preschool children with ADHD and DBD: a 18 month-longitudinal study. Developmental Neuropsychology, 39(4), 302-315.

Weiner, I.B. (2000). Making Rorschach interpretation as good as it can be. Journal of Personality Assessment, 74(2), 164-174.

Weiner, I. (2003). Principles of Rorschach interpretation. New York: Routledge.

Yáñez-Téllez, G., Romero-Romero, H., Rivera-García, L., Prieto-Corona, B., Bernal-Hernández, J., Marosi-Holczberger, E., Guerrero-Juárez, V., Rodríguez-Camacho, M., y Silva-Pereyra, J. (2012). Cognitive and executive functions in ADHD. Actas Españolas de Psiquiatría, 40(6), 293-298. 